\title{
Malignant hyperpyrexia: a rare cause of postoperative death
}

\author{
R J Prescott, S P Roberts, G Williams
}

\begin{abstract}
A middle aged man developed very high fever, status epilepticus, and terminal acute renal failure with myoglobinuria after surgery. A post mortem examination showed widespread muscle necrosis with hypercontraction bands. Muscle enzyme studies and electron microscopic examination disclosed central core disease, a condition closely related to malignant hyperpyrexia. This condition is a genetically inherited disorder which can be triggered by certain volatile anaesthetic agents or Suxamethonium. In this patient the condition may have been triggered by either the Isoflurane or the postoperative status epilepticus.
\end{abstract}

Department of

Christie Hospital and

Holt Radium Institute,

Withington,

Manchester M20 9EX

R J Prescott

Department of

Anaesthesia, Stepping

Hill Hospital,

Stockport

$S$ P Roberts

Department of

Pathology, University

of Manchester

G Williams

Correspondence to:

Dr R J Prescott

Accepted for publication

5 September 1991

\section{Case report}

A 50 year old British man underwent craniotomy for excision of a tuberculum sella meningioma. He had no past or family history of anaesthetic problems. The anaesthesia used was Thiopentone, Atracurium, Fentanyl, nitrous oxide and Isoflurane, rather than Halothane. Suxamethonium was not given. His vital signs were normal, and he had no fever on return to the ward.

Two hours after recovery, he had a single Phenytoin. Two hours later he went into status epilepticus which lasted for one hour. He had a persistent tachycardia of 107 beats a minute, a temperature of $42^{\circ} \mathrm{C}$, and a generalised seizure which settled with metabolic acidosis (arterial blood pH of 7·1).

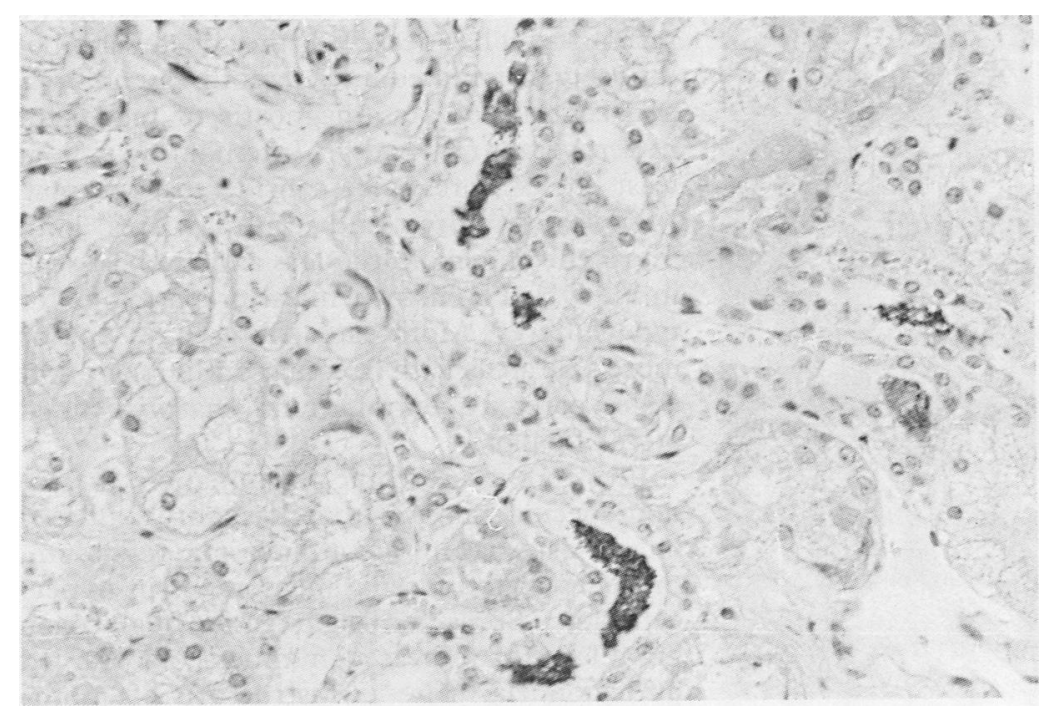

Figure 1 Renal tubular casts showing strong positive staining with antisera against myoglobin protein (immunoperoxidase).
A computed tomography scan showed right frontal lobe contusion and haemorrhage with brain shift; this was considered to be secondary to the surgical procedure. A blood clot was removed during which he was given Suxamethonium. He remained feverish, acidotic, and hypokalaemic (serum $\mathrm{K}^{+}=$ $2.32 \mathrm{mmol} / 1$ ).

The following day he developed acute renal failure associated with myoglobinuria. His neurological signs warranted limited frontal lobectomy, he became hypotensive, and died.

\section{Pathology}

The meningioma measured $1 \mathrm{~cm}$ in diameter and had a typical meningotheliomatous pattern without malignant features. Post mortem examination of the brain showed haemorrhage and contusions in the residual right fronto-parietal lobe. There was no evidence of brain shift or coning-related phenomena. Histological examination disclosed no pathological abnormalities in the non-traumatised areas, in particular the hypothalamus which contains the temperature regulating centres.

The kidneys were swollen and pale, each weighing in excess of $200 \mathrm{~g}$. The corticomedullary interface was indistinct and there was bleeding in the pelvicalyceal system. Histological examination showed acute tubular necrosis of both proximal and distal tubules associated with numerous, deeply eosinophilic casts. The latter stained strongly with antisera to myoglobin, using a standard immunoperoxidase technique (fig 1 ). The vasa recta contained nucleated red blood cells, related to the terminal hypotension.

Sections of striated muscle from several anatomical sites showed extensive segmental necrosis and hypercontraction bands, associated with very little, if any, inflammatory response. No regenerative features were present, indicating rapid, acute rhabdomyolysis. The viable areas showed variation in myofibre diameter, sarcoplasmic vacuolation and internalisation of nuclei (fig 2).

These appearances were identical with those of an antemortem quadriceps muscle biopsy which also disclosed central core disease, selectively affecting $64 \%$ of type I fibres. This was shown by standard histochemical techniques using nicotinamide adenosine diaphorase-tetrazolium reductase (NADH-TR) and adenosine triphosphatase enzymes at various $\mathrm{pH}$ of $9 \cdot 4,4 \cdot 6$, and $4 \cdot 2$ (fig 3). Electron microscopic examination 


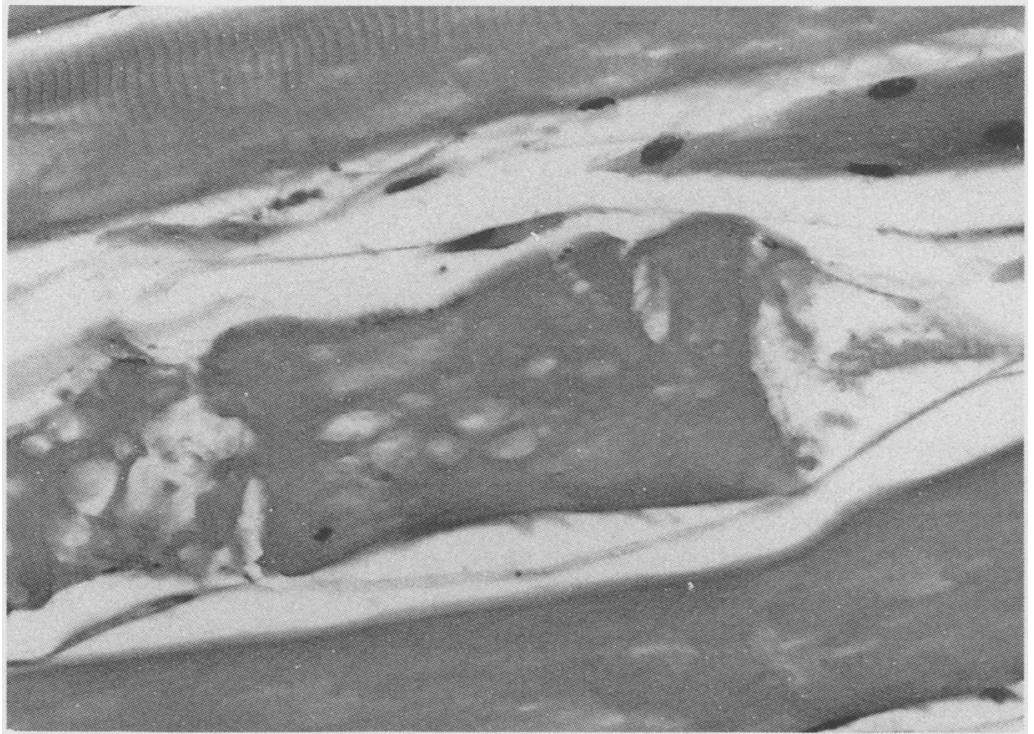

Figure 2 Striated muscle showing necrosis and internalisation of nuclei (haematoxylin and eosin).

confirmed the presence of cores devoid of mitochondria and myofilaments.

Given these findings, combined with the clinicobiochemical derangements, malignant hyperpyrexia was diagnosed. The mechanism of death was considered to be prolonged rhabdomyolysis resulting in myoglobinuria and acute tubular necrosis. The myoglobinuria might have been related to the hyperpyrexia, status epilepticus, or the operation. An intracranial cause for the observed hyperpyrexia cannot totally be excluded even in the absence of any histological abnormality in the brain, particularly the hypothalamus. Given the muscle central core disease and the presence of two possible triggering events (the administration of Isoflurane and status epilepticus), it is more likely that the underlying mode of death was related to the malignant hyperpyrexia.

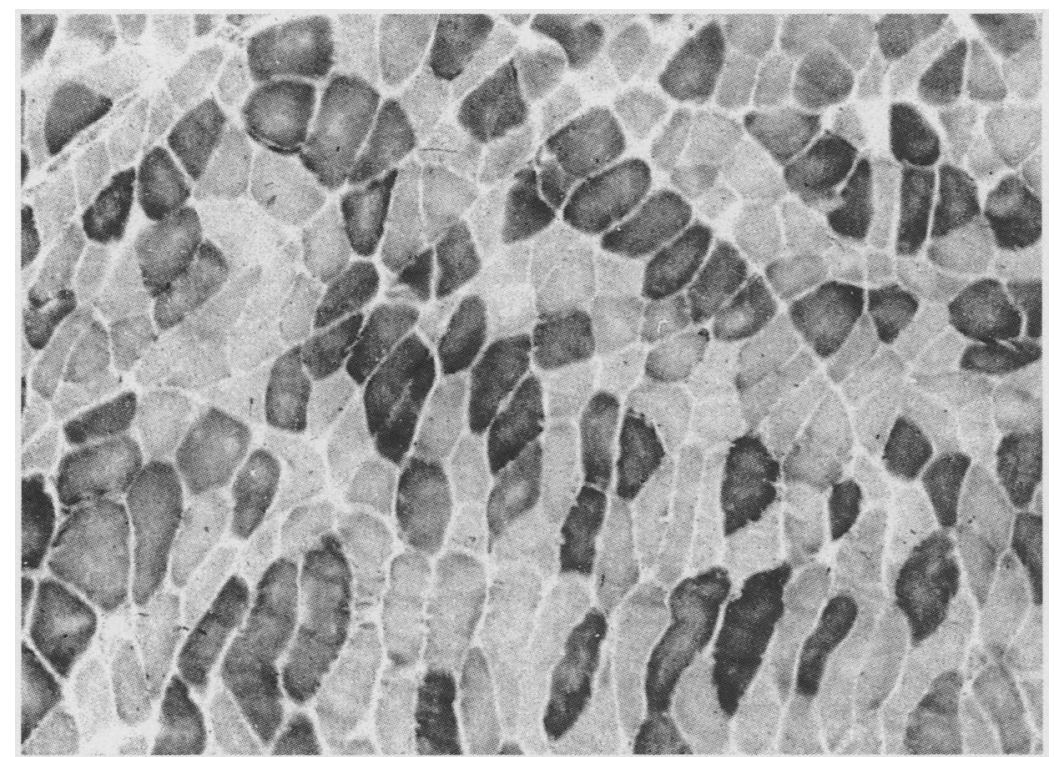

Figure 3 Histochemical appearance of quadriceps muscle, showing central core defects in the darker stained type I fibres (NADH-TR).

\section{Discussion}

Malignant hyperpyrexia is an autosomal dominant inherited condition usually presenting postoperatively with a progressive rise in body temperature at a rate of $2-6^{\circ} \mathrm{C}$ per hour. ${ }^{1}$ It was first described in $1960 .^{2}$ The incidence in the United Kingdom has been quoted as 1 in 200000 and the male:female ratio as $2: 1{ }^{1}$

Malignant hyperpyrexia is triggered by the neuromuscular junction blocking agent Suxamethonium and by volatile anaesthetic agents; Halothane is the commonest agent described but Isoflurane has also been implicated. ${ }^{34}$ Ingestion of large amounts of ethanol and conditions of physical stress including trauma and status epilepticus are also triggering stimuli. ${ }^{1}$

The underlying abnormality has been attributed to a genetically determined, latent, molecular defect residing in the sarcoplasmic reticulum. There is either decreased sequestration of calcium in the sarcoplasmic reticulum or increased release, or both. This defect is aggravated by the aforementioned exogenous agents. The sustained release of calcium ions produces hypercontraction of muscle fibres, necrosis, and liberation of myoglobin, potassium ions, lactic acid and enzymes including creatine phosphokinase (CPK). The mechanism of the temperature rise is not well understood but it seems that the hypermetabolic state acts as a positive feedback loop causing the temperature and metabolism to increase further. Late recognition and severity of disease account for a mortality around $50 \%$ for all cases. ${ }^{1}$

Screening of relatives is an important aspect of malignant hyperpyrexia and includes muscle biopsy. The demonstration of increased contracture response to caffeine or a caffeine/ Halothane mixture of in vitro fresh muscle is diagnostic.

Histological examination of antemortem muscle may show no abnormality, or a wide range of non-specific changes, including variation in fibre diameter, fibre splitting, atrophic fibres, central nuclei, nuclear chains, tubular aggregates and sarcoplasmic vacuolation. ${ }^{5}$ Despite this spectrum of reported changes, a recent study has described the constellation of diffusely distributed nuclei, moth-eaten fibres, and central cores as diagnostic of malignant hyperpyrexia myopathy. ${ }^{6}$ Internal nuclei in at least $3 \%$ of fibres were considered abnormal. Moth-eaten fibres and central cores both represent mitochondrial loss and are best seen in NADH-TR histochemical preparations for type I fibres. Moth-eaten fibres have one or more pale areas with irregular outlines and soft edges; cores are central, round, sharply defined areas of pallor.

Electron microscopic examination is also non-specific, ranging from normality to dilated lateral cisternae in the sarcoplasmic reticulum, breaches in the plasma membranes in hypercontracted fibres, focal aggregation of glycogen granules and increased numbers of enlarged mitochondria. The ultrastructure of motheaten fibres and central cores shows loss of mitochondria and occasional fibrils displaying 
limited Z-band streaming, a feature related to severe contraction of individual myofibrils.

The association of malignant hyperpyrexia with central core disease is well established, ${ }^{7}$ so much so, that all patients with this condition, including the relatives, should be considered to be at risk from malignant hyperpyrexia unless in vitro contracture tests indicate otherwise.

The association with Duchenne's muscular dystrophy and other myopathies is less definite but it does lend support to the concept that the, as yet unidentified, malignant hyperpyrexia gene is probably located near to the other myopathy related genes.
We thank Mr A Strang, consultant neurosurgeon, for allowing this case to be reported, and the Medical Illustration Department, Manchester Medical School, for the histological photography.

1 Ellis FR, Halsall PJ. Malignant hyperpyrexia. $\mathrm{Br} J$ Hosp Med 1980;24:318-27.

2 Denborough MA, Lovell RRH. Anaesthetic deaths in a family. Lancet $1960 ; \mathrm{ii}: 45$.

3 Joseph MM, Shah K, Vilioen JF. Malignant hyperthermia associated with Isoflurane anesthesia. Anesth Analges associated with

4 Boheler J, Eger EI. Isoflurane and malignant hyperthermia. Anesth Analges 1982;61:712-13.

5 Harriman DGF. Malignant hyperthermia myopathy-a critical review. Br J Anaesth 1988;60:309-16.

6 Harriman DGF, Sumner DW, Ellis FR. Malignant hyperpyrexia myopathy. $Q J$ Med 1973;42:639-64.

7 Brownell AKW. Malignant hyperthermia: relationship to other diseases. Br J Anaesth 1988;60:303-8.

\title{
Dieulafoy's vascular malformation as a cause of large intestinal bleeding
}

\author{
D J Farrell, M K Bennett
}

\begin{abstract}
Four cases of Dieulafoy's vascular malformation of the caecum are reported. Three were associated with massive large bowel haemorrhage, one of which was fatal. The remaining case had a calibre persistent submucosal artery within the caecum that was found incidentally in a resection specimen.

This vascular malformation should be considered when dealing with specimens resected for massive lower gastrointestinal bleeding.
\end{abstract}

Dieulafoy's vascular malformation, also known as calibre persistent submucosal artery, is a rare but well known cause of upper gastrointestinal bleeding. ${ }^{1}$ It has been found almost exclusively in the stomach, although cases in the duodenum ${ }^{2}$ and jejunum ${ }^{3}$ have also been reported. Recently it has been recognised that it may occur in the colon and can cause massive bleeding. ${ }^{4}$ To date there have been six cases of large bowel haemorrhage from a Dieulafoy type ulcer. $^{4-7}$ We report four more cases.

\section{Case reports}

CASE 1

Department of Histopathology, Freeman Hospital, High Heaton, Newcastle upon Tyne NE7 7DN D J Farrell M K Bennett Correspondence to: Dr D J Farrell Accepted for publication 5 September 1991
A 77 year old man was admitted with severe continuous bleeding from the rectum and lower abdominal pain which he had had for six hours. He had previously undergone repair of a perforated gastric ulcer and was known to have polycythemia rubra vera. He was in circulatory shock and required transfusion of 8 units of blood over the following 10 hours. An emergency oesophago-gastro-duodenoscopic examination was normal. Because of continued bleeding, emergency superior mesenteric angiography was performed which showed arterial bleeding in the caecal tip. An emergency right hemicolectomy was therefore carried out. At surgery blood had filled the whole of the large bowel but there seemed to be none in the small bowel.

After inflation of the specimen with barium to show the vessels, macroscopic examination showed a small ulcer $0.2 \mathrm{~cm}$ in diameter inside the tip of the caecum. Microscopic examination of the caecal ulcer showed a thrombus covering a muscular artery of large diameter within the submucosa and extending into the mucosa which had ruptured into the lumen (fig 1). There was no evidence of arteritis. There was a localised inflammatory response in the immediately adjacent mucosa but the bowel was otherwise unremarkable. The appearances were identical with those of a Dieulafoy ulcer as described in the stomach. ${ }^{1}$

\section{CASE 2}

A 64 year old man with known alcoholic liver disease was admitted with anorexia, weight loss, increasing dyspnoea and melaena. On examination he had the stigmata of chronic liver disease. Because of repeated nose bleeds he required multiple transfusions but continued to be anaemic. He was also known to have oesophageal varices. He continued to pass large volumes of melaena with blood clots daily and had evidence of a chest infection. Despite supportive measures he died eight days after admission.

Post mortem examination showed a widespread, diffuse non-Hodgkin's lymphoma. Altered blood was present in the stomach and 